\section{ACUTE MEDICINE}

This edition explores the increasing variety of clinical challenges from acute medicine. Articles on subjects ranging from asthma to diving medicine, gastroenteritis to $\beta$ blocker overdose, ECG artefact to accuracy of death certification should stimulate thought and hopefully be relevant to your clinical practice.

\section{ACUTE BACTERIAL GASTROENTERITIS}

Gastroenteritis is not one of the most fashionable areas of emergency medicine. Therefore the study by Chan et al is useful in highlighting this condition. The authors point out that the incidence of causative agents will vary in different countries and even within a community. However, they provoke interesting discussion on the utility of stool culture and of antibiotic treatment. What are your department's guidelines?

See page 335

\section{INTRAVENOUS THERAPY FOR CHOLERA}

The article by Foex on the history of intravenous therapy for cholera is a scholarly review of one of the most important advances in medical treatment of fluid loss. A valuable reflection on how a treatment we now regard as routine, came into use.

See page 316

\section{ACCURACY OF DEATH CERTIFICATION}

Sudden death in the emergency department is a traumatic event and it can be difficult to discuss the need for a postmortem examination with bereaved relatives. Often we rely on the decision of the coroner (or procurator fiscal) on the need for a postmortem examination.
However, the study by Quigley and Burton points out that in up to two thirds of cases the evidence for the certified cause of death may be flimsy. The postmortem examination is a powerful audit and learning tool, especially in the context of unexpected death. However, many of the patients dying in A\&E are elderly, often as the result of the end stage of chronic diseases processes. We have to judge whether the information gleaned from a postmortem examination will help or hinder the bereavement process. The article highlights the importance of discussing all cases of sudden unexpected death with no clear cause with the coroner (or procurator fiscal). See page 349

\section{ACUTE ASTHMA}

Acute asthma is one of those high volume medical emergencies with well recognised management pathways. This audit of the documentation of vital signs in patients with acute asthma reveals both good and bad news. The rates of recording heart rate, respiratory rate, and oxygen saturation are high but the recording of peak expiratory flow rate remains disappointingly low. While this is another audit of process with no evidence of a link with outcome, all would agree that we should be recording peak flow rates as a key measure of the severity of the episode and to gauge the response to treatment.

See page 329

\section{SHOCKS FROM IMPLANTED DEFIBRILLATORS}

There have always been small but significant risks to the health of clinical staff treating patients. The current outbreak of sudden acute respiratory syndrome is a reminder of the dangers of infectious disease. However, it seems that modern technology has added a new risk, the possibility of being shocked by implanted automatic defibrillators. The authors state that the risks can be much reduced by wearing gloves and the defibrillator can be disabled by a magnet. With the National Institute for Clinical Excellence recommending a large increase in the use of this treatment you should be checking on where the magnets are kept in your department.

See page 379

\section{D-DIMER TESTING}

One of the largest areas of research in emergency medicine is the search for the holy grail of the test that will "rule out" common clinical conditions. The article by Wakia et al reviews the confused area of D-dimer. It is clear that the literature is complex because of the multiplicity of techniques used for D-dimer estimation. The early promise of an easy near patient test for thromboembolic disease has not been realised. However, this article collates the evidence that D-dimer will have a part to play in the future but a great deal of work needs to be done to define the best test to use in the population of patients attending our departments. An increasing number of emergency medicine departments are implementing "rule out" strategies for deep venous thrombosis or pulmonary embolism and this would seem an area ripe for multicentre collaboration.

See page 319 